\title{
Problematización de las prácticas docentes y contextualización de la enseñanza
}

\section{Problematization of Teaching Practices and Teaching Contextualization}

Jorge Rodríguez-Sosa *1 (iD Universidad San Ignacio de Loyola, Lima, Perú. ORCID: https://orcid.org/0000-0002-8440-4891

Kelly Hernández-Sánchez 2 (D) Universidad San Ignacio de Loyola, Lima, Perú. ORCID: https://orcid.org/0000-0003-2793-9678

${ }^{1}$ Magister en Sociología. Docente investigador de la Dirección de Estudios Generales de la Universidad San Ignacio de Loyola. ${ }^{2}$ Licenciada en Ciencias de la Comunicación. Coordinadora de Cursos CPEL y Programas Complementarios de la Dirección de Estudios Generales de la Universidad San Ignacio de Loyola.

Recibido 21-02-18 Revisado 28-02-18 Aprobado 07-05-18 En línea 08-05-18

*Correspondencia

Email: jrodriguezs@usil.edu.pe

\section{Citar como:}

Rodríguez-Sosa, J., \& Hernández-Sánchez, K. (2018). Problematización de las prácticas docentes y contextualización de la enseñanza. Propósitos y Representaciones, 6(1), 507-541. doi: http://dx.doi. org/10.20511/pyr2018.v6n1.211

(c) Universidad San Ignacio de Loyola, Vicerrectorado de Investigación, 2018

(cc) BY-NC-ND Este artículo se distribuye bajo licencia CC BY-NC-ND 4.0 Internacional (http://creativecommons.org/licenses/by-nc-nd/4.0/). 


\section{Resumen}

El artículo es una reflexión en tres planos: el primero, sobre cómo los docentes problematizan sus propias prácticas dada la complejidad de los escenarios que deben enfrentar cotidianamente en las aulas. El segundo, sobre cómo la problematización de la docencia en percibida en el mundo académico y el escolar. El tercero, sobre cuánto y cómo los contenidos, las herramientas metodológicas y los formatos de capacitación, que son necesarios para fortalecer en los docentes las capacidades y las actitudes proclives a la problematización, están siendo asumidos por los programas de formación docente continua. Se concluye con una reflexión conjunta que trata de establecer el vínculo entre los planos.

Palabras clave: Problematización de las prácticas docentes, investigación de la docencia, investigación-acción, programas de formación docente continua, creencias docentes.

\section{Summary}

This article is a reflection in three planes: the first one, how teachers problematize their own practices given the complexity of the scenarios that they must face daily in classrooms; the second one, how teaching problematization is perceived in the academic and school world and the third one, hoy many and how contents, methodological tools and training forms, which are necessary to strengthen teachers' abilities and attitudes prone to problematization, have been assumed by continuous teaching training programs. It is concluded with a joint reflection that tries to establish the link between these planes.

Keywords: Problematization of teaching practices, teaching research, research-action, continuous teaching training programs, teacher's beliefs. 


\section{Introducción}

Los docentes Latinoamericanas de hoy enfrentan escenarios de alta complejidad en el marco de sus prácticas cotidianas en aula. Desde la academia se exige que las escuelas implementen programas de inclusión y una enseñanza que atienda la diversidad (López, 2009). Desde la política se les exige que alcancen logros mínimos en accesos, aprendizajes y permanencias (Fernandes, 2014), por lo menos equivalentes a los que las escuelas alcanzan en otras partes del mundo. Desde la sociedad, aprendizajes relevantes y contextualizados, principalmente en el campo de las ciencias (Herrington, Yezierski, Luxford \& Luxford, 2011), más una oferta con servicios de calidad (Aranguren, 2007). Se trata de exigencias distintas pero convergentes que deben ser atendidas en entornos sociales caracterizados por la desigualdad, el individualismo y manifestaciones crecientes de violencia.

Estos escenarios están ostensiblemente alejados de aquellos desde los cuales y para los cuales se definieron las escuelas hace tres o cuatro décadas. Los cambios que han ocurrido entre unos escenarios y otros han tornando a la docencia en una actividad poco predecible, incierta y compleja (Perrenoud, 2004). Paralelamente, han ensanchado las brechas entre lo que las escuelas están en capacidad de dar y las necesidades emergentes de los estudiantes. Los docentes aparecen consecuentemente como operadores inermes de procesos que no pueden gestionar (López, 2009),

El perfil del docente que las escuelas de hoy necesitan es el de un profesional orientado hacia una práctica reflexiva y contextual (Chacón, Chacón \& Alcedo, 2012). Debe incluir un conjunto de capacidades y herramientas que son necesarias para que los docentes puedan elaborar propuestas de enseñanza que aseguren un mínimo de ajuste entre las ofertas institucionales escolares y las necesidades locales (Rodríguez-Sosa, CáceresCruz \& Rivera-Gavilano, 2017). Las políticas de formación continua de docentes tienen este reto y muchas tareas pendientes. 


\section{¿Cómo los docentes problematizan sus prácticas?}

Los ejercicios y testimonios que presentamos en este segmento corresponden a un grupo de 21 docentes en servicio participantes del Programa de Especialización en Tutoría y Acompañamiento Pedagógico del Ministerio de Educación del Perú (MINEDU), ejecutado por la Facultad de Educación de la Universidad Peruana Cayetano Heredia. Las reflexiones sobre esos ejercicios y testimonios son nuestras, resultaron de nuestra experiencia de participación como equipo técnico en el componente de investigación-acción implementado por la Facultad en el marco del Programa de Especialización mencionado. Una versión más amplia y profunda de esta experiencia, que incluye buena parte de estos ejercicios y testimonios, así como las reflexiones que se hizo sobre ellos, fue expuesta en el artículo Experiencia de innovación en desarrollo profesional docente (Rodríguez-Sosa, Lingán, Hernández \& Alhuay-Quispe, 2017).

El Programa de Especialización tuvo como propósito certificar a los 21 docentes como monitores de docentes en aula, quienes posteriormente cumplirían esas funciones con grupos más extensos de docentes en ejercicio, como parte del Programa Nacional de Formación y Capacitación Pedagógica (PRONAFCAP), programa público masivo de capacitación de docentes también del MINEDU. El componente de investigación-acción fue insertado en el Programa de Especialización y comprendió tres módulos ejecutados en 10 semanas. En total 120 horas, 48 de estudios presenciales y 72 de estudio independiente. Los estudios presenciales se dieron en dos formatos, uno de talleres para la presentación de los contenidos, procedimientos y casos vinculados a los temas que abordó cada módulo, el otro, los círculos de reflexión, para la discusión abierta sobre aquellos temas tocados en los talleres que se consideraron especialmente relevantes. El propósito del componente fue que los docentes entendiesen la importancia de incorporar la reflexión crítica y una actitud de auto-inspección de sus prácticas. También se esperaba que, en ese marco, se explicitasen y cuestionasen las creencias 
que traían, aquellas que daban sustento a lo que hacían, favoreciendo la exploración de nuevas maneras de concebir y de hacer las cosas.

Los testimonios de los docentes fueron recogidos en grabaciones hechas de los círculos de reflexión y las sesiones de taller. Una primera ruta de análisis de los testimonios fue longitudinal. Permitió comparar "con qué opiniones entraron" y "con qué opiniones salieron" los docentes, para establecer los cambios que experimentaron por efectos de su participación en el componente. Una segunda ruta de análisis trabajó sobre las convergencias y divergencias en las percepciones sobre los hechos. En ambas rutas se empleó matrices de doble entrada: en la entrada vertical los docentes y en la horizontal los testimonios con los temas abordados. Sobre esa masa de información se separó aquellos testimonios que no eran de utilidad, para luego aplicar los procedimientos convencionales del análisis cualitativo: reducción de datos, presentación de datos e interpretación (Miles \& Huberman, 1984).

Los ejercicios metodológicos fueron parte de los productos exigidos en cada módulo del componente y trabajados en grupos de tres docentes. Se trató de ejercicios que se brindaron con el propósito de fortalecer y de evaluar habilidades en la formulación de problemas, la identificación de sus causas, la justificación de su abordaje y el planteamiento de una propuesta de solución, así como habilidades para el empleo de evidencias e información. Se partió del supuesto de que estos ejercicios exponían la comprensión que los docentes alcanzaban de la investigación en general y de la problematización de la docencia en particular. Fueron evaluados en términos de su pertinencia, relevancia y fundamentación, entendiendo que un problema es pertinente cuando es identificable en las prácticas docentes cotidianas y puede ser abordado desde la práctica misma (Rodríguez Sosa, 2005), es relevante cuando se justifica por la potencial introducción de beneficios (y de beneficiarios) y/o por sus implicancias prácticas en la solución de problemas (Hernández, Fernández-Collado \& Baptista, 2007), y está fundamentado cuando se basa en evidencia recogida de la investigación en el campo (Slafer, 2009). 
La investigación previa reporta que los docentes tienen muy poca o ninguna disposición hacia la auto-inspección de sus prácticas (GonzalezWeil, Cortez, Pérez, Bravo \& Ibaceta, 2013). Los testimonios recogidos de los 21 docentes muestran que perciben los problemas que se manifiestan en sus aulas como hechos cuyos orígenes deben ser buscados en ámbitos externos a las prácticas docentes. Algunos de los ámbitos recurrentes son los rasgos de personalidad de los estudiantes o supuestas limitaciones en sus capacidades. En relación a lo primero, se mencionó a jóvenes que tienen muy poca motivación por el estudio, o no tienen ninguna, o niños que no tienen curiosidad por algún tema, que no son curiosos, no intentan descubrir [...] su desinterés es en todo. En cuanto a lo segundo, se mencionó que: es dificil que puedan tener algún aprendizaje si comprenden la mitad de lo que leen o menos, entonces cómo esperamos que entiendan lo que se les pide resolver en un problema. Otro ámbito recurrente fue el de los problemas en las familias: este muchacho está condenado por la pobreza de su familia [...] puede tener capacidad para el estudio pero lo importante en su familia es que trabaje y traiga algún ingreso adicional a la casa.

Con esa concepción los docentes identifican problemas que, con algunas variaciones, tienen un sentido similar al siguiente: manifestaciones de desorden y bullying entre los estudiantes cuando se trabaja con espacios no convencionales (colectivos, participativos, lúdicos) en el aula. Las causas que estarían asociadas a este problema son: los entornos sociales violentos, la violencia cotidiana que ha permeado la escuela o los altos niveles de desestructuración en las familias que no favorecen la transmisión de reglas de convivencia, ni el respeto a normas básicas, con similares niveles de incidencia.

Como se puede ver, se trata de un abordaje sesgado del problema que busca las causas del mismo en un "otro" externo a lo que ocurre en el aula (características que trae el estudiante, problemas en sus familias, entorno social de la escuela), a quien se le atribuye la capacidad de influir en lo que el docente puede hacer y que estaría explicando los pobres desempeños o 
cualquier otro déficit, mientras en simultáneo se evita la necesaria reflexión sobre las propias prácticas, ejercicio que podría llevar a que los docentes ubiquen los problemas en lo que hacen (o dejan de hacer) en el aula. Adicionalmente, cuando se hizo el ejercicio de anticipar algunas respuestas a los problemas identificados (o hipótesis de acción, si se quiere), se mantuvo la búsqueda en ámbitos externos a las prácticas. Como resultado de ello, las soluciones propuestas se debían procesar fuera de la escuela, incluso bajo la responsabilidad de actores distintos a los actores escolares. Ese abordaje evitaba el conocimiento de las situaciones reales a las que el docente debía enfrentar, limitando de paso la posibilidad de anticipar estrategias de solución efectivas, pues se operaba frente a problemas que no existían en la escuela o que se percibían muy distorsionados.

La situación se fue revirtiendo progresivamente conforme avanzaba la ejecución del componente, especialmente en los espacios brindados por los círculos de reflexión, que abrieron excelentes oportunidades para la reflexión colectiva y el diálogo abierto. Sin embargo, este proceso de cambios no fue fluido sino más bien resistido. Lo primero que se advirtió fue que si bien los docentes aceptaban que reflexionar sistemáticamente era importante, también entendían que se trataba de un proceso complejo que suponía introducir cambios en unas maneras de hacer las cosas en las que creen y para las que tienen las destrezas necesarias. Por ello, pese a que los cambios buscados no se dieron en todos los docentes, ni se alcanzaron con las mismas intensidades o en los mismos plazos, en la mayoría de los casos las concepciones transitaron del tenemos este problema con causas que tienen su origen fuera de la escuela, pero que sin embargo limita lo que podemos hacer como docentes, a un acercamiento distinto que se cuestionaba sobre qué cambios en las maneras como hacemos las cosas (porque no hemos tenido la disposición o las capacidades necesarias) supone el abordar efectivamente ese tipo de problemas. Este nuevo enfoque ubicaba inmediatamente a los problemas en el ámbito de las prácticas en aula, restituyendo intencionalidad pedagógica a las soluciones y protagonismo a los docentes. 
Así el problema identificado, presentado párrafos arriba, fue abordado desde una perspectiva distinta y definido como la necesidad de consensuar y explicitar actividades, normas y acuerdos con los estudiantes para aprovechar al máximo los espacios no convencionales del trabajo en aula (colectivos, participativos, lúdicos). Las causas de este nuevo problema fueron buscadas en aquello que el docente hace: carencias en el manejo de metodologías para el trabajo en grupos, el poco conocimiento de dinámicas que favorezcan la participación de los estudiantes, o no se cuenta con herramientas suficientes para despertar interés y motivación. Los nuevos abordajes muestran que los docentes encontraron los factores explicativos y las posibilidades de solución de los problemas en zonas cada vez más cercanas a sus ámbitos de influencia, en sus prácticas. También sugieren que en lo actitudinal los docentes asumieron posturas proclives a la auto-inspección de esas mismas prácticas.

La propuesta re-definida (o mejor dicho re-hecha, con nueva orientación) supuso un cambio radical, pues se pasó de un enfoque donde el rol del docente no era activo: manifestaciones de desorden y bullying entre los estudiantes cuando se trabaja con espacios no convencionales (colectivos, participativos, lúdicos) en el aula, a otro en que la solución era parte de la actividad del docente. Asimismo, las causas del problema fueron ubicadas en la práctica misma, identificándolas como carencias que el docente tenía en el manejo de herramientas para el trabajo en grupos, la participación de los estudiantes o la motivación. De ese modo, las estrategias de intervención tendrían que enfocarse en el fortalecimiento de esas capacidades: la implementación de un taller en didácticas participativas con docentes; el rediseño organizacional del aula, previendo un espacio de diálogo, acuerdos y toma de decisiones; $y$ darle capacidades de decisión a los estudiantes para seleccionar los temas clave que serán abordados desde los espacios no convencionales.

Esos cambios muestran que los docentes dejaron de ver a los problemas como situaciones que vienen "desde fuera" de la escuela, para pasar a entenderlos como desajustes entre aquello que vienen haciendo y lo que se 
requiere hacer, dadas las características y necesidades de los grupos a los que se atiende. Con ello, los docentes no solo recuperaron la capacidad de intervenir en la mejora de sus prácticas, puesto que ubicaron los problemas en su zona de influencia, sino que ampliaron sus posibilidades de intervención con soluciones que al ejecutarse debían producir en simultáneo mejoras en la enseñanza.

\section{Las percepciones de la investigación (y de la problematización de las prácticas docentes) desde el mundo académico y desde la escuela}

La teoría crítica en pedagogía sostiene que la investigación educativa es tal si en su ejercicio se valora las capacidades de los docentes de producir conocimiento pedagógico -además de involucrarlos como protagonistas en los procesos-, y se tiene como finalidad la mejora permanente de sus prácticas (Elliot, 2010; McKernan, 1996; Carr \& Kemmis, 1988). Se trata de un paradigma que integra a la investigación con el ejercicio de la docencia y el aprendizaje profesional (Zeichner, 1993).

Si llevásemos estos principios de la teoría crítica al plano de un programa real de investigación, estaríamos hablando de un tipo diferente de estudios que requerirían de un nuevo investigador, uno colectivo e inmerso en las situaciones sobre las que problematiza, que las observa desde su propia perspectiva y que complementa la metodología con los saberes docentes, buscando con ello generar nuevas comprensiones sobre las prácticas desde el lugar en que estas prácticas ocurren (Montecinos, Solis \& Gabriele, 2001). Esta nueva situación no excluiría al investigador académico pero si limitaría su participación al propósito de fortalecer las iniciativas de los docentes, quiénes tendrían el protagonismo en las distintas fases del proceso: en la identificación de problemas (aquellos que los docentes consideren como los más sentidos y urgentes), en la selección de estrategias de intervención (aquellas que se consideren pertinentes y viables), en la evaluación de lo hecho (las valoraciones y re-significaciones), y en la institucionalización de los cambios (Rodríguez-Sosa, 2005). 
En las últimas tres décadas, este paradigma de la pedagogía crítica se ha encumbrado en como el paradigma hegemónico en el discurso de los programas de formación docente en Latinaomérica, mientras en la práctica real estos programas continúan encasillados en los modelos tradicionales (Messina, 1999). En un sentido similar, la investigación educativa real Latinoamericana no ha recorrido el camino propuesto por la investigación desde la práctica, sino que continúa sujeta a los estándares de la investigación académica convencional.

Es probable que la poca valoración que los investigadores del mundo académico universitario tienen de aquellas investigaciones que emplean metodologías participativas y análisis cualitativos, explique buena parte de la separación entre la investigación académica y el sistema de formación de docentes (Molinari \& Ruiz, 2009). Como resultado, la investigación ha tenido una presencia excepcional en la escuela, que además se dio como una práctica que vino "desde fuera" sin establecer articulaciones con la docencia (Imbernón, 2007). Una investigación principalmente a cargo de investigadores académicos, profesionales foráneos al mundo de la escuela (psicólogos, sociólogos, economistas, entre otros), que observaron temas cuya elección dependía de sus perspectivas e intereses particulares, dejando por fuera y sin reflexión sistemática el extenso campo de las prácticas de enseñanza (Molinari \& Ruiz, 2009).

En simultáneo -o quizás explicado en buena cuenta por ello-, los docentes conciben la problematización de sus propias prácticas como un hecho puntual, distinto y externo a la enseñanza, que eventualmente podría sumarse a lo que hacen de haber una justificación para ello. La auto-inspección de lo que se hace no es percibida como una necesidad, menos como un componente esencial en la enseñanza. La disposición a sumar elementos de problematización en la enseñanza es muy pobre, más si esas adiciones suponen cambiar varios de los procesos que son parte de las prácticas.

Algunas de las opiniones que dieron los docentes al inicio del componente exponen razones vinculadas a su escepticismo sobre el aporte 
que la problematización de las prácticas podría significar en la mejora de la docencia: Si uno no tiene los conocimientos de lo que va a enseñar o no maneja las metodologías necesarias, no va a enseñar bien, por más que sea consciente de los problemas que tiene, de sus limitaciones [...] Creo que eso es lo principal, luego uno puede plantearse cuestionamientos y retos, o razones que dan prioridad a la transmisión de contenidos: Un conocimiento cercano de los problemas que los estudiantes puedan tener es importante, pero la prioridad son los contenidos [...] En el empleo de un tiempo escaso elijo desarrollar todos mis contenidos y hacerlo bien.

Sin embargo, esas opiniones fueron cambiando, al menos parcialmente, aunque desde ellas no fuese posible prever la introducción de algún nivel de cambios en las prácticas: Yo empleo la problematización. Hablo de mí no de mis colegas. Yo me cuestiono, identifico problemas, trabas, "cuellos de botella" que les dicen en la docencia que hago. Siempre trato de hacer las cosas mejor, busco soluciones y me planteo retos. Claro, lo hago informalmente, fuera del trabajo, cuando estoy en mi casa [...] Creo que tiene alguna utilidad [...] Uno hace estas cosas [problematizar] si es consciente, si está comprometido con la docencia. No sé si los colegas lo hacen, seguro que algunos sí, pero ni se cómo lo hacen. Seguro que cada quien tiene su método. Los testimonios recogidos en este punto permiten inferir que a los docentes no les fue posible presentar ideas generales sobre cómo insertar la problematización en la docencia, sea a nivel de las didácticas, la programación de los contenidos, o en la mejora de las actividades o los materiales de trabajo.

\section{La necesidad de nuevos enfoques en la formación continua de docentes}

Diversos estudios en América Latina reportan sobre realidades donde los diseños curriculares están fragmentados y mantienen una escasa vinculación con los contextos escolares donde los docentes llevan a cabo sus prácticas (Vaillant, 2009; Rodríguez, 2004). Este desencuentro entre lo que las escuelas 
quieren o pueden hacer -expresado en los currículos-, y las necesidades locales es una de las manifestaciones de las brechas existentes entre una formación inicial de docentes pensada para otros escenarios, una formación en servicio alineada y complementaria a la anterior, y las necesidades que hoy tienen las escuelas reales. Por supuesto, la existencia de estas brechas, así como la necesidad de cerrarlas en el corto plazo, es una de las principales preocupaciones de los gobiernos en materia de formación docente (OECD, 2005).

En respuesta a ello, los estados latinoamericanos han venido cediendo progresivamente sus monopolios en los programas de formación continua de docentes, abriéndose a la participación de nuevos operadores como universidades y otras instituciones (ONG, institutos de formación, grupos de reflexión), que trajeron consigo un discurso que promovía el empleo de metodologías participativas y de programas de empoderamiento de actores locales (Flores-Kastanis, Montoya-Vargas \& Suárez, 2009). Sin embargo, lejos de lo esperado los nuevos operadores no integraron en sus programas los cambios declarados como necesarios en lo pedagógico u organizativo, por el contrario mantuvieron los esquemas tradicionales, aquellos limitados al fortalecimiento de contenidos disciplinares $\mathrm{y} / \mathrm{o}$ de metodologías didácticas, con el empleo de enfoques remediales y lógicas prescriptivas (Fernandes, 2014; González-Weil, et. al., 2013). La capacitación no tuvo ni tiene como prioridad el fortalecimiento de los docentes en las capacidades y herramientas necesarias para afrontar las situaciones (problemas, conflictos, dilemas) que ocurren en el aula, en especial si lo que se busca es responder a las necesidades de grupos diversos con una enseñanza contextualizada (Montero, 2011).

Fullan (2002; pp. 122) indica con ironía que "la educación del profesorado tiene el honor de ser, al mismo tiempo, el peor problema y la mejor solución de la educación". En otras palabras, la sola capacitación no asegura que luego los docentes plasmen lo aprendido introduciendo cambios en sus prácticas y menos que lo hagan en la dirección esperada. La adopción 
de nuevas maneras de pensar y de hacer las cosas necesitan de una base de creencias alineada con esas orientaciones, como una condición que de soporte a la disposición de los docentes a cambiar (Herrington, et. al., 2011). La literatura en el campo reporta que el cambio de las creencias docentes, que por naturaleza son implícitas y no conscientes, sólo es posible en el marco de procesos que favorezcan su explicitación (Pozo, Scheuer, Mateos \& Pérez, 2006). En ese sentido, los procesos de capacitación tendrían los efectos necesarios si consideran operar con espacios donde los docentes puedan cuestionar sus propias creencias, desde perspectivas reflexivas, abiertas y auto-críticas, como una condición organizacional indispensable (Chamizo \& Garcia-Franco, 2013; Park Rogers, et al., 2007; Vezub, 2007).

Ibernón (2001) sostiene que la formación de docentes más adecuada es aquella que se da en colectivos que emplean sistemáticamente la reflexión deliberativa en el marco metodológico de la investigación-acción. En el mismo sentido, una revisión documental de experiencias de este tipo hecha por Zeichner (2005), además de otros estudios puntuales (Chacón, Chacón \& Alcedo, 2012; Yamin-Ali, 2010; Herrington et al., 2011; MegovanRomanowicz, 2010; Ruiz-Mallen, Barraza, Bodenhorn, de la Paz CejaAdame \& Reyes-García, 2010; Blanchard, Southerland \& Granger, 2009; Maarof, 2007), reportan hallazgos que vinculan a la investigación-acción con los cambios en la enseñanza.

\section{Conclusiones}

La experiencia del componente de investigación-acción en el marco del Programa de Especialización permitió observar que los docentes cambiaron radicalmente sus percepciones sobre los problemas propios de sus prácticas, pasando de entenderlos como situaciones que vienen desde fuera de la escuela a asumirlos como aquello que es necesario cambiar en lo que se hace, con el propósito de alinear la enseñanza con las necesidades de los grupos a los que se atiende. Los docentes recuperaron las capacidades de intervenir en sus prácticas al situar los problemas en su zona de influencia 
y vincular las soluciones a la introducción de mejoras en la enseñanza. Incluso las propuestas de intervención mismas pasaron a ser entendidas como procesos de aprendizaje y de desarrollo profesional, mientras el empleo de la investigación de la docencia en apoyo de esos procesos de fortalecimiento empezó a tener sentido para el docente mismo. Estos cambios son consistentes con lo reportado en experiencias que emplearon metodologías de investigación-acción en el fortalecimiento de las habilidades de identificación y exploración de problemas en el aula (Yamin-Ali, 2010), o en el desarrollo del pensamiento reflexivo del docente con fines de mejora de sus prácticas (Halim, Buang \& Meerah, 2010).

Otro ámbito importante de cambios reportado por la experiencia fue el referido a cómo enseñar, a qué dar prioridad y cómo administrar los tiempos disponibles para ello. Los testimonios dan cuenta de movimientos desde una concepción de la docencia que tiene a la transmisión de contenidos como fin esencial, hacia una concepción que valora el hecho de evaluar los niveles de efectividad de distintas maneras de enseñar. Lo importante de estos cambios es que las concepciones mencionadas representan la manera como los docentes construyen sus modelos de enseñanza deseable y dan soporte a la toma de decisiones.

Finalmente, la experiencia del componente nos muestra que las capacitaciones tendrían que ser entendidas como procesos formativos continuos que persiguen cambios en las prácticas docentes, entendiendo que alcanzarlos supondría pasar necesariamente por cambios previos en las creencias, percepciones y actitudes sobre la enseñanza que los docentes tienen, a la vez que situar a la enseñanza como un objeto constante de problematización (Rodríguez-Sosa, 2005). No se trataría de una formación estocástica y alejada (alienada) de la práctica, sino de una formación permanente que se produciría en el contexto de la propia práctica. 


\section{Referencias}

Aranguren, G. (2007). Systemizing action research as an intervention and teacher training strategy in its research role. Revista de Pedagogía, 28(82), 173-195.

Blanchard, M., Southerland, S., \& Granger, E. (2009). No silver bullet for inquiry: making sense of teacher change following an inquiry based research experience for teachers, Science Education, 93(2), 322-360. Doi: https://doi.org/10.1002/sce.20298

Carr, W., \& Kemmis, S. (1988). Teoría crítica de la enseñanza. La investigación acción en la formación del profesorado. Barcelona: Editorial Martínez Roca.

Chacón, MA., Chacón, C., \& Alcedo, Y. (2012). Los proyectos de aprendizaje interdisciplinarios en la formación docente. Revista Mexicana de Investigación Educativa, 17(54), 877-902.

Chamizo, JA. \& García-Franco, A. (2013). Heuristics diagrams as a tool to formatively assess teachers' research". Teachers and Teaching: theory and practice, 19(2), 135-149. Doi: http://dx.doi.org/10.1080/13540602 .2013 .741841

Elliot, J. (2010). La investigación acción en educación. Madrid: Morata.

Fernandes, C. (2014). Relaciones entre la investigación y la formación docente permanente: El conocimiento necesario para la diversidad. Estudios Pedagógicos, 40(2)161-174. http://dx.doi.org/10.4067/S071807052014000300010

Flores-Kastanis, E., Montoya-Vargas, J., \& Suárez, D. (2009). Investigaciónacción participativa en la educación Latinoamericana. Un mapa de otra parte del mundo. Revista Mexicana de Investigación Educativa, 14(40), 289-308.

Fullan, M. (2002). Las fuerzas del cambio. Explorando las profundidades de la reforma educativa. Madrid: Akal.

González-Weil, C., Cortez, M., Pérez, JL., Bravo, P., \& Ibaceta, Y. (2013). Construyendo dominios de encuentro para problematizar acerca de las prácticas pedagógicas de profesores secundarios de Ciencias: Incorporando el modelo de Investigación-Acción como plan de 
formación continua. Estudios pedagógicos, 39(2), 129-146. Doi: http:// dx.doi.org/10.4067/S0718-07052013000200009

Halim, L., Buang, NA., \& Meerah, S. (2010). “Action research as instructional supervision: Impact on the profesional development of university based supervisors and science student teachers". Procedia. Social and Vihavioral Sciences, 2(2), 2868-2871. Doi: http://dx.doi.org/10.1016/j. sbspro.2010.03.430

Hernández, R., Fernández-Collado, C., \& Baptista, P. (2007). Metodología de la investigación. México: McGraw-Hill / Interamericana.

Herrington, D., Yezierski, E., Luxford, K., \& Luxford, C. (2011). Target inquiry: Changing chemistry high school teachers' classroom practices and knowledge and beliefs about inquiry instruction". Chemistry Education Research and Practice, 33(1), 74-84. Doi: http://dx.doi. org/10.1039/C1RP90010B

Imbernón, F. (2001). Training in schools: Trend or fashion? In F. López (Comp.), Teacher Training: Training projects in schools (pp. 15-22). Barcelona: Grao.

Imbernón, F. (2007). La investigación educativa como herramienta de formación del profesorado. Barcelona: Grao.

López, N. (coordinador). (2009). De relaciones, actores y territorios. Hacia nuevas políticas para la educación en América Latina. Buenos Aires: IIPE-UNESCO.

Maarof, N. (2007). Telling his or her story through reflective journals. International Education Journal, 8(1), 205-220. Recuperado de: http:// ehlt.flinders.edu.au/education/iej/articles/v8n1/Maarof/paper.pdf

McCkernan, J. (1996). Investigación-acción y currículo. Madrid: Morata.

Megowan-Romanowicz, C. (2010). Inside out: Action research from the teacher-researcher perspective". Journal of Science Teacher Education, 21(8), 993-1011. Doi: http://dx.doi.org/10.1007/s10972-010-9214-z

Messina, G. (1999). Investigación en o investigación acerca de la formación docente: un estado del arte en los noventa. Revista Iberoamericana de Educación. 19, 145-207 
Miles, M., \& Huberman, M. (1984). Qualitative data analysis. A source book of new methods. Beverly Hills: Sage.

Molinari, A., \& Ruiz, G. (2009). Consideraciones acerca de la investigación en las instituciones de formación de profesores. Archivos de Ciencias de la Educación, 3(3), 129-140. Recuperado de: http://www. archivosdeciencias.fahce.unlp.edu.ar/article/view/ARCHv03n03a10

Montecinos, C., Solis, MC. \& Gabriele, A. (2001). "Aprendiendo a enseñar a través de la investigación-acción". Paideia Revista de Educación, vol. 30, núm. 31, pp. 37-50.

Montero,C.(2011).Estudio sobre acompañamientopedagógico.Experiencias, orientaciones y temas pendientes". En: Consejo Nacional de Educación. Hacia una propuesta de criterios de buen desempeño docente. Estudios que aportan a la reflexión, al diálogo y a la construcción concertada de una política educativa. Lima: Consejo Nacional de Educación, pp. 71172 .

Organisation for Economic Co-operation and Development. (2005). Attracting, developing and retaining effective teachers. Final Report: Teachers matter. Paris: OECD.

Park Rogers, M., Abell, S., Lannin, J., Wang, Ch., Musikul, K., Barker, D. \& Dingman, S. (2007). "Effective professional development in science and mathematics education: teachers' and facilitators' views". International Journal of Science and Mathematics Education, 5(3), 507-532. Doi: http://dx.doi.org/10.1007/s10763-006-9053-8

Perrenoud, P. (2004). Developing reflective practice. Barcelona: Grao.

Pozo, J., Scheuer, N., Mateos, M. \& Pérez, M. (2006). Las teorías implícitas sobre el aprendizaje y la enseñanz", en Pozo, J., Scheuer, N., Pérez, M., Mateos, M., Martín, E. \& De La Cruz, M. (2006). Nuevas formas de pensar la enseñanza y el aprendizaje, Barcelona: Grao. pp. 95-132.

Rodríguez, N. (2004). Challenges of teacher training in Venezuela. Revista de Pedagogía, 25(73), 03-12.

Rodríguez-Sosa, J. (2005). La investigación acción educativa ¿Qué es? ¿Cómo se hace? Lima: Doxa. 
Rodríguez-Sosa, J., Lingán, S., Hernández, R., \& Alhuay, J. (2017). Experiencia de innovación en desarrollo profesional docente. Interdisciplinaria, 34(2), 407-424. Recuperado de: http://www.ciipme-conicet.gov.ar/ojs/index. $\mathrm{php} /$ interdisciplinaria/article/view/347

Rodríguez-Sosa, J., Cáceres-Cruz, M., \& Rivera-Gavilano, P. (2017) New scenarios in latin american schools. The needs for a contextualized teaching practice. Propósitos y Representaciones, 5(2), 15-19. Doi: https:// doi.org/10.20511/pyr2017.v5n2.193

Ruiz Mallen, I., Barraza, L., Bodenhorn, B., Ceja Adame, M., \& Reyes García, V. (2010). Contextualising learning through the participatory construction of an environmental education programme". International journal of science education, 32(13), 1755-1770. Doi: http://dx.doi. org/10.1080/09500690903203135

Slafer, G. (2009). ¿Cómo escribir un artículo científico?. Revista de Investigación en Educación, núm. 6, pp. 124-132.

Vaillant, D. (2009). Secondary school teachers training: Realities and speeches. Revista de Educación, 350, 105-122. Recuperado de: http:// www.revistaeducacion.mec.es/re350/re350_05.pdf

Vezub, L. (2007). La formación y el desarrollo profesional docente frente a los nuevos desafíos de la escolaridad". Profesorado. Revista de Currículum y Formación de Profesorado, 11(1), 1-23. Recuperado de: http://recyt.fecyt.es/index.php/profesorado/article/view/41837

Yamin-Ali, J. (2010). Translating concerns into action in the foreign language classroom. GEMA Online Journal of Language Studies, 11(2), 21-38. Recuperado de: http://ejournal.ukm.my/gema/article/view/54

Zeichner, K. (1993). El maestro como profesional reflexivo. Cuadernos de pedagogía, 220, 44-49.

Zeichner, K. (2005). Educational Action Research. En: Reason. Peter y Bradbury-Huang, Hilary (eds.). Handbook of Action Research. Participative inquiry and practice. London: Sage. 